\title{
EVALUATION OF RATIONAL FUNCTION MODEL FOR GEOMETRIC MODELING OF CHANG'E-1 CCD IMAGES
}

\author{
Yiliang Liu, Kaichang Di* \\ Institute of Remote Sensing Applications, Chinese Academy of Sciences \\ P. O. Box 9718, Datun Rd, Chaoyang District, Beijing 100101, P.R.China \\ (ylliu, kcdi)@irsa.ac.cn
}

Commission IV, WG IV/7

KEY WORDS: Planetary, Mapping, Geometric, Model, Rational Function Model, Chang'E-1, CCD image, Precision

\begin{abstract}
:
Rational Function Model (RFM) is a generic geometric model that has been widely used in geometric processing of high-resolution earth-observation satellite images, due to its generality and excellent capability of fitting complex rigorous sensor models. In this paper, the feasibility and precision of RFM for geometric modeling of China's Chang'E-1 (CE-1) lunar orbiter images is presented. The RFM parameters of forward-, nadir- and backward-looking CE-1 images are generated though least squares solution using virtual control points derived from the rigorous sensor model. The precision of the RFM is evaluated by comparing with the rigorous sensor model in both image space and object space. Experimental results using nine images from three orbits show that RFM can precisely fit the rigorous sensor model of CE-1 CCD images with a RMS residual error of 1/100 pixel level in image space and less than 5 meters in object space. This indicates that it is feasible to use RFM to describe the imaging geometry of CE-1 CCD images and spacecraft position and orientation. RFM will enable planetary data centers to have an option to supply RFM parameters of orbital images while keeping the original orbit trajectory data confidential.
\end{abstract}

\section{INTRODUCTION}

Chang'E-1(CE-1) orbiter, the first lunar probe of China, was launched on October 24, 2007. It left lunar transfer orbit on 31 October and entered a $200 \mathrm{~km}$ altitude circular lunar orbit on November 5. CE-1 successfully completed its mission by crashing onto the Moon surface on March 1, 2009. During the exploration period, the CCD camera onboard CE-1 acquired more than 1000 imagery strips which provided complete coverage of the Moon (Ouyang et al., 2010; Li et al., 2010). A 1:2.5 million scale global image mosaic of the Moon has been produced using the CCD images after radiometric and geometric processing, map projection, mosaicking and editing (Li et al., 2010).

The CE-1 CCD camera is a three line pushbroom camera which has a ground resolution of $120 \mathrm{~m}$ and a swath width of $60 \mathrm{~km}$. In order to perform 3D mapping from the stereo images, the geometric model of the images should be established firstly. We have developed a rigorous sensor model for CE-1 based on pushbroom imaging principle and the exterior orientation parameters derived from spacecraft trajectory (position and orientation) data (Peng et al., 2010). Methods of automatic DEM and orthoimage generation, and co-registration of CE-1 CCD images and laser altimeter (LAM) data, were also developed (Di et al., 2010). However, the spacecraft trajectory data of CE-1 is not open to public so far, though the CCD images and other scientific data have been released for public access.

Rational Function Model (RFM) is one of the generic geometric models in photogrammetry and remote sensing to represent the transformation between image space and object space. Due to its generality and excellent capability of fitting complex rigorous sensor models, it has been widely used in geometric

processing of high-resolution earth-observation satellite images, especially when the rigorous sensor models are not supplied and internal geometric parameters are not disclosed (Di et al., 2001). So far, to the best of our knowledge, no study on feasibility and application of FRM for extra-terrestrial mapping (such as lunar and Mars mapping) has been reported.

In this paper, we investigate the feasibility and precision of fitting rigorous sensor model with RFM using CE-1 CCD images. At first, the rigorous sensor model is briefly introduced. Then the process of generating rational polynomial coefficients (RPCs) is described. In the process, vast amount of virtual control points are generated based on the rigorous sensor model, the RPCs are solved iteratively using a least squares estimation. Finally, the precision of the RFM is evaluated by comparing with the rigorous sensor model in both image space and object space.

\section{RIGOROUS GEOMETRIC MODEL OF CE-1 CCD IMAGERY}

\subsection{Interior Orientation}

The CE-1 CCD camera is a three line pushbroom camera which

\footnotetext{
*Correspding author
} 
is implemented on an area array CCD sensor $(1024 \times 1024$ pixels). The actual imaging area is 1024 rows by 512 columns. The focal length of the CCD camera is $23.33 \mathrm{~mm}$. While imaging, it uses only the 11th, 512th and 1013th rows to generate the forward-, nadir- and backward-looking images simultaneously in the flight direction (Li et al., 2010). The structure of the focal plane of the CE-1 CCD camera is shown in Figure 1.

Interior orientation refers to the transformation from the image coordinates (columns and rows) to their focal plane coordinates centered at the principal point of the image according to the calibrated camera interior orientation parameters. The focal plane coordinates can be calculated from image coordinates by Equation (1) (Peng et al., 2010):

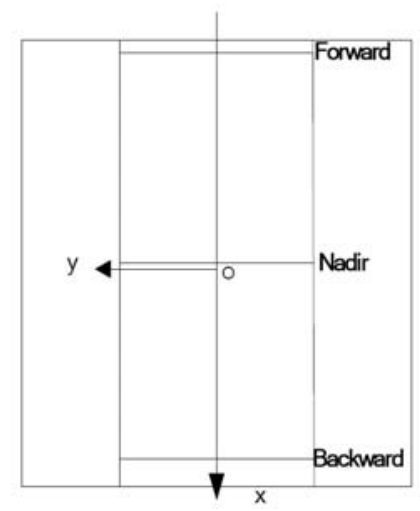

Figure 1. Focal plane frame of the CE-1 CCD camera

$$
\begin{aligned}
& x_{\text {forward }}=\left(x_{p}-11\right) * \text { pixsize }-x_{0} \\
& x_{\text {nadir }}=\left(x_{p}-512\right) * \text { pixsize }-x_{0} \\
& x_{\text {back }}=\left(x_{p}-1013\right) * \text { pixsize }-x_{0} \\
& y_{\text {forward }}=y_{\text {nadir }}=y_{\text {back }}=\left(y_{p}-\text { col }\right) * \text { pixsize }-y_{0}
\end{aligned}
$$

where pixsize stands for the pixel size of the CCD array, which is $0.014 \mathrm{~mm}$; col is the pixel position in column direction; $\left(x_{p}\right.$, $\left.y_{p}\right)$ are the center position $(511.5,255.5)$ of the actual imaging area; $\left(x_{0}, y_{0}\right)$ represent principal point position in the focal plane frame; $\left(x_{\text {forward }}, y_{\text {forward }}\right),\left(x_{\text {nadir }}, y_{\text {nadir }}\right)$ and $\left(x_{\text {back }}, y_{\text {back }}\right)$ are focal plane coordinates of forward-, nadir-, backward-looking images respectively.

\subsection{Exterior Orientation}

Exterior orientation refers to the transformation between the focal plane frame and lunar body-fixed frame (LBF). It requires the exterior orientations parameters (EOPs). Combining interior orientation and exterior orientation, the rigorous sensor model of CE-1 CCD imagery can be represented as the following collinearity equations:

$$
\begin{aligned}
& x=-f \frac{m_{11}\left(X-X_{O}\right)+m_{12}\left(Y-Y_{O}\right)+m_{13}\left(Z-Z_{O}\right)}{m_{31}\left(X-X_{O}\right)+m_{32}\left(Y-Y_{O}\right)+m_{33}\left(Z-Z_{O}\right)} \\
& y=-f \frac{m_{21}\left(X-X_{O}\right)+m_{22}\left(Y-Y_{O}\right)+m_{23}\left(Z-Z_{O}\right)}{m_{31}\left(X-X_{O}\right)+m_{32}\left(Y-Y_{O}\right)+m_{33}\left(Z-Z_{O}\right)}
\end{aligned}
$$

where $(x, y)$ are focal plane coordinates of an image point; $(X, Y$, $Z$ ) are the corresponding 3D coordinates in LBF; $m_{\mathrm{ij}}(\mathrm{i}, \mathrm{j}=1,2,3)$ are the elements of the rotation matrix determined by three Euler angles $\omega, \phi, \kappa ;\left(X_{O}, Y_{O}, Z_{O}\right)$ are the camera center position in LBF. $\left(X_{O}, Y_{O}, Z_{O}, \omega, \phi, \kappa\right)$ are called EOPs. For pushbroom sensors, each image line has a specific set of EOPs.

The original telemetry EOPs of CE-1 were collected at $1 \mathrm{~Hz}$ frequency, which is much lower than the frequency of the CCD camera scanning. Therefore, interpolation of telemetry EOPs is necessary to obtain the EOPs of every scan line. In this study, Lagrange interpolating polynomial is used.

Given a set of $k+1$ data points, for example, $\left(x_{0}, y_{0}\right), \ldots$, $\left(x_{j}, y_{j}\right), \ldots,\left(x_{k}, y_{k}\right)$, where no two $x_{j}$ are the same, the interpolation polynomial in the Lagrange form is a linear combination as

$$
L(x)=\sum_{j=0}^{k} y_{j} a_{j}(x)
$$

of Lagrange basis polynomials

$$
\mathrm{a}_{j}(x)=\prod_{\substack{0 \leq m \leq k \\ m \neq j}} \frac{x-x_{m}}{x_{j}-x_{m}}
$$

Apparently, all basis polynomials are zero at $x=x_{i}$ except $a_{i}(x)=1$, because it lacks the $x-x_{i}$ term. It follows that $y_{i} \mathrm{a}_{i}\left(x_{i}\right)=y_{i}$, so at each point $x_{i}, L\left(x_{i}\right)=y_{i}$, showing that $L$ interpolates the function exactly (Wikipedia, 2011).

In our experiment, Lagrange polynomial can fit a curve exactly through the known original telemetry data. Through the interpolation, the EOPs of each image lines of forward-, nadir-, backward-looking images are obtained. Consequently, the rigorous sensor models are established for the images.

\subsection{Space Intersection and Back-projection}

Based on the rigorous sensor model and the known interior and exterior orientation parameters of the image scan lines, space intersection and back-projection can be implemented easily. Given a 3D ground point, the corresponding image point can be directly calculated by back-projection using the collinearity equations. Given the conjugate points in forward-, nadir-, backward-looking images, the corresponding 3D ground points can be calculated by space intersection, i.e., least squares solution of ground points using linearized collinearity equations.

Image matching is a key technique to find conjugate image points from stereo images. We have developed a multi-level image matching method in this study. After image preprocessing, such as Gaussian filtering and histogram normalization, a SIFT algorithm (Lowe, 2004) is applied for feature point extraction and matching. Then, a RANSAC procedure (Fischler and Boolles, 1981) is performed to 
eliminate matching outliers (gross errors). Next, a triangulated irregular network (TIN)-controlled dense image matching method is adopted for dense grid matching (Peng et al., 2010) and hundreds of thousands of conjugate points are generated. Based on these dense points, 3D ground point can be calculated through space interception. Consequently, DEM can be generated by spatial interpolation using these dense points.

\section{RATIONAL FUNCTION MODEL OF CE-1 CCD IMAGERY}

\subsection{Rational Function Model}

In the RFM, image coordinates $(x, y)$ are expressed as a ratio of two polynomials which contain ground coordinates $(X, Y, Z)$ as shown in Equation 5.

$$
\begin{aligned}
& x=\frac{P_{1}(X, Y, Z)}{P_{2}(X, Y, Z)} \\
& y=\frac{P_{3}(X, Y, Z)}{P_{4}(X, Y, Z)}
\end{aligned}
$$

Polynomials $P_{i}(i=1,2,3$, and 4$)$ have the general form as

$$
P(X, Y, Z)=\sum_{i=0}^{m_{1}} \sum_{j=0}^{m_{2}} \sum_{k=0}^{m_{3}} a_{i j k} X^{i} Y^{j} Z^{k}
$$

where $m_{1}, m_{2}, m_{3}$ and the sum of them are limited by [0,3], so as to describe $P(X, Y, Z)$ as a three-order, 20-term polynomial. Plugging Equation (6) into Equation (5) with them, we can get the final expression of RFM as follows (Di, 2001):

$$
\begin{aligned}
& x=\frac{\left(1 X Y Z \cdots Y Z^{2} Z^{3}\right)\left(a_{0} a_{1} a_{2} a_{3} \cdots a_{18} a_{19}\right)^{T}}{\left(1 X Y Z \cdots Y Z^{2} Z^{3}\right)\left(1 b_{1} b_{2} b_{3} \cdots b_{18} b_{19}\right)^{T}} \\
& y=\frac{\left(1 X Y Z \cdots Y Z^{2} Z^{3}\right)\left(c_{0} c_{1} c_{2} c_{3} \cdots c_{18} c_{19}\right)^{T}}{\left(1 X Y Z \cdots Y Z^{2} Z^{3}\right)\left(1 d_{1} d_{2} d_{3} \cdots d_{18} d_{19}\right)^{T}}
\end{aligned}
$$

It should be noted that image coordinates $(x, y)$ and ground coordinates $(X, Y, Z)$ should be normalized to the range from 1.0 to 1.0 by their image size and geometric extent, respectively, for computational stability and minimizing computational errors (Di et al., 2001).

\subsection{Rational Polynomial Coefficients (RPCs) Generation}

Generation of RFM parameters, i.e., RPCs, of the CE-1 CCD imagery is basically a process of fitting the rigorous sensor model with RFM. It is realized by solving RPCs in Equation 7 using vast number of virtual control points generated from the rigorous sensor model. First, grid points are generated in image space with a certain interval in $x$ and $y$ directions. Then we slice the $\mathrm{Z}$ direction into several layers in object space and use the rigorous sensor model (Equation 2) to generate corresponding virtual ground points.

With known corresponding image and ground coordinates of the virtual control points, we can solve the RPCs in Equation 7 by least squares principle. Since RFM equations are nonlinear, they are firstly linearized using Taylor's theorem. The linearized observation equation can be represented as

$$
V=A C-L
$$

The corresponding least squares solution is

$$
C=\left(A^{T} A\right)^{-1} A^{T} L
$$

Note that $C$ is the unknown vector that includes all RPCs. Equation 8 could be ill-posed because of the high order (i.e., $3^{\text {rd }}$ order) polynomials. To avoid possible ill condition, Tikhonov regularization is incorporated in the solution. The solution equation becomes

$$
C=\left(A^{T} A+h^{2} I\right)^{-1} A^{T} L
$$

where $h$ is the regularization parameter and I is the identity matrix.

\subsection{Space Intersection and Back-projection with RFM}

In a similar way of the rigorous sensor model, RFM can be used for space intersection to calculate 3D ground positions from conjugate images points in stereo images. Given the RPCs of the stereo images, image coordinates of conjugate points, and the initial approximations of the ground position, the leastsquares solution of the ground position is obtained iteratively using linearized FRM equations (Di et al., 2001). For CE-1 CCD images, any two of the forward-, nadir-, backwardlooking images can form a stereo pair for space intersection. Using 3 images together in space intersection gives best accuracy because of redundant observations. Back-projection is straightforward. Given the RPCs of an image and the 3D coordinates of a ground point, the corresponding image point can be directly calculated using Equation 7 .

\section{EXPERIMENTAL RESULTS}

Rainbow bay area is one of the pre-selected landing sites for the upcoming CE-3 lunar rover. CE-1 CCD images covering this area (centered at $46^{\circ} \mathrm{N}, 30^{\circ} \mathrm{W}$ ) from three orbits $(0561,0562$ and 0563 ) are used in our experiment. The images of the three orbits were acquired on December 25-26, 2007 and each image of the whole orbit was taken in about fifty minutes. Each orbit includes forward-, nadir-, backward-looking CCD images covering from the South Pole to the North Pole. Figure 2 shows the forward-, nadir-, backward-looking images acquired from orbit 0562 (partial, 2000 lines).

In the experiment, we use 10,000 rows of the forward-, nadir-, backward-looking images of the three orbits to check the fitting precision of FRM. More than 15,000 virtual control points are generated with seven layers in $\mathrm{Z}$ direction, and then RPCs for each image is generated through least squares solution. The root 
mean square (RMS) residuals of the fitting are listed Table 1. The results show that the FRM can fit the rigorous model very well with a precision of $1 / 100$ pixel level in image space
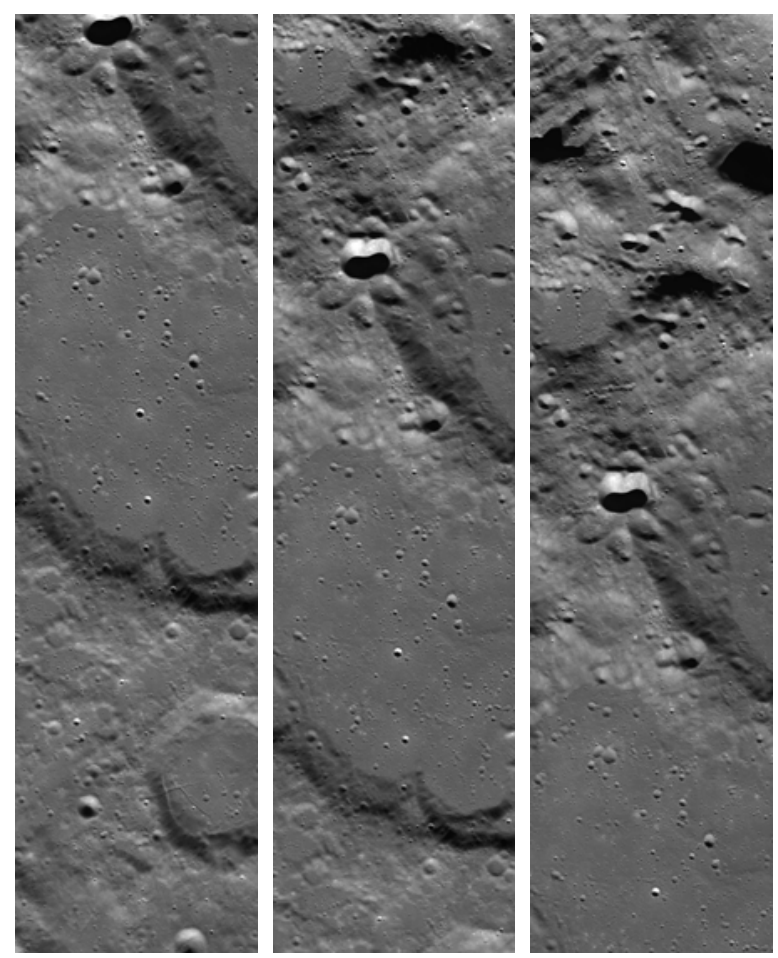

(a) Forward-looking (b) Nadir-looking (c) Backward-looking Figure 2. Partial forward-, nadir-, and backward-looking lunar images taken by CE-1 from orbit 0562

\begin{tabular}{|c|c|c|c|}
\hline $\begin{array}{c}\text { Orbit } \\
\text { Number }\end{array}$ & Image & $\begin{array}{c}\text { RMS x } \\
\text { (pixel) }\end{array}$ & $\begin{array}{c}\text { RMS y } \\
\text { (pixel) }\end{array}$ \\
\hline \multirow{3}{*}{0561} & Forward-looking & 0.02 & 0.02 \\
\cline { 2 - 4 } & Nadir-looking & 0.01 & 0.02 \\
\cline { 2 - 4 } & Backward-looking & 0.02 & 0.04 \\
\hline \multirow{3}{*}{0562} & Forward-looking & 0.02 & 0.02 \\
\cline { 2 - 4 } & Nadir-looking & 0.01 & 0.02 \\
\cline { 2 - 4 } & Backward-looking & 0.02 & 0.04 \\
\hline \multirow{3}{*}{0563} & Forward-looking & 0.02 & 0.02 \\
\cline { 2 - 4 } & Nadir-looking & 0.00 & 0.02 \\
\cline { 2 - 4 } & Backward-looking & 0.02 & 0.04 \\
\hline
\end{tabular}

Table 1. FRM fitting precision in image space

\begin{tabular}{|l|l|l|l|}
\hline $\begin{array}{l}\text { Orbit } \\
\text { Number }\end{array}$ & $\begin{array}{l}\text { X-RMS } \\
(\mathrm{m})\end{array}$ & $\begin{array}{l}\text { Y-RMS } \\
(\mathrm{m})\end{array}$ & $\begin{array}{l}\text { Z-RMS } \\
(\mathrm{m})\end{array}$ \\
\hline 0561 & 2.80 & 1.74 & 4.08 \\
\hline 0562 & 2.73 & 1.65 & 4.10 \\
\hline 0563 & 2.77 & 1.69 & 4.28 \\
\hline
\end{tabular}

Table 2. RFM precision in object space

In order to evaluate the RFM precision in object space, more than 150,000 coordinates of ground points are generated from matched conjugate points in forward-, nadir-, backward-looking images by space intersection with RPCs and then are compared with those generated with the rigorous sensor model. As shown in Table 2, RMS differences are less than 5 meters.

Considering the relatively low space resolution of $120 \mathrm{~m}$, the RFM fitting precision is satisfactory in both image and object spaces. From the above two tables, we can draw a conclusion that the attitude of CE-1 lunar probe is stable enough and FRM can be used to describe the imaging geometry of CE-1 CCD imagery with an accuracy comparable to the rigorous sensor model..

\section{SUMMARY AND DISCUSSION}

Comparing with rigorous sensor model, RFM provides a generic and simpler model for geometric processing of orbital imagery and will enable planetary data centers to have an option to supply RPCs of orbital images while keeping the original orbit trajectory data confidential. In this paper, we investigated the feasibility and precision of fitting rigorous sensor model with RFM using CE-1 CCD images. Experimental results indicate that RFM can precisely fit the rigorous sensor model of CE-1 CCD images with a RMS residual error of 1/100 pixel level in image space and less than 5 meters in object space. More comprehensive tests will be performed using more lunar and Mars orbital images to confirm the feasibility of FRM for geometric processing of planetary images.

\section{ACKNOWLEDGEMENTS}

Funding of this research by National Natural Science Foundation of China $(40871202,41002120)$ and the National High Technology Research and Development Program of China (2009AA12Z310) is acknowledged. We thank the Lunar and Deep Space Exploration Science Applications Center of the National Astronomical Observatories (NAOC) for providing the CE-1 CCD image data.

\section{REFERENCES}

Di, K., Ma, R., Li, R., 2001. Deriving 3-D shorelines from high resolution IKONOS satellite images with rational functions. ASPRS (American Society for Photogrammetry \& Remote Sensing) Annual Conference. St. Louis, MO.

Di. K., Yue. Z., Peng. M., and Liu, Z., 2010. Co-registration of CHANG'E-1 stereo images and laser altimeter data for 3D mapping of lunar surface. ASPRS/CaGIS 2010 Specialty Conference, Orlando, Florida, USA, December 15-19.

Fischler, M.A., Boolles, R.C., 1981. Random Sample consensus: a paradigm for model fitting with applications to image and automated cartography. Graphics and Image Processing, 24(6), pp.381-395.

Mathews, J.H., Fink, K.D., 2002. Numerical Methods Using MATLAB (Third Edition), Trans. Chen, Y., et al., Publishing House of Electronics Industry, Beijing, pp. 154-164.

Li, C., Liu, J., Ren, X., et al., 2010. The global image of the Moon obtained by the Chang'E-1 Data processing and lunar cartography. Sci China Earth Sci, 40(3), pp.294-306.

Lowe, D. G., 2004. Distinctive Image Features from ScaleInvariant Keypoints. International Journal of Computer Vision, 
International Archives of the Photogrammetry, Remote Sensing and Spatial Information Sciences, Volume XXXVIII-4/W25, 2011 ISPRS Guilin 2011 Workshop, 20-21 October 2011, Guilin, China

60(2), pp. 91-110.

Ouyang, Z., Li, C., Zou, Y., et al., 2010. The Primary Science Results from the Chang'e-1 Probe. Sci China Earth Sci, 40(3), pp.261-280.
Peng, M., Yue, Z., Liu, Y., Di, K., 2010. Research on lunar and Mars orbital stereo image mapping. The 17th Remote Sensing Conference of China, Hangzhou, China.

Wikipedia, 2011. Lagrange polynomial, http://en.wikipedia.org/wiki/Lagrange_polynomial (accessed 27 June 2011) 